\title{
Sporotrichoid Cutaneous Leishmaniasis in Central Tunisia: Epidemiological and Clinical Aspects
}

\author{
Moncef Ben Saïd, Fatma Saghrouni, \\ Yusr Saadi-Ben Aoun, Najet Ghariani, Alia Yaacoub, \\ Henda Ach, Mohamed Denguezli, \\ Ikram Guizani and Akila Fathallah-Mili
}

Additional information is available at the end of the chapter

http://dx.doi.org/10.5772/57413

\section{Introduction}

Three forms of cutaneous leishmaniasis (CL) are known to occur in Tunisia: the sporadic CL (SCL), the chronic CL (CCL) and the zoonotic CL (ZCL) caused by Leishmania infantum, L. killicki and L. major respectively [1,2]. The ZCL is by far the most frequent and the most widely distributed form. It is endemo-epidemic in extended areas of central and southern Tunisia where it constitutes a major public health problem. Rodents of Genus Psammomys and Meriones are the well-established reservoirs of the disease [1, 2]. One of the characteristics of ZCL is its seasonal occurrence as most cases are diagnosed between October and January [1]. All ages are affected with a median of 20-28 years. Clinically, ZCL usually presents as single or most often multiple ulcerated lesions that involve the face and limbs, and scar in a few months (4 to 8) [3]. However, some patients with ZCL develop sporotrichoid nodules (SN) in the vicinity of the primary lesions $[4,5,6]$. The sporotrichoid condition is characterized by the development of nodules that progress, starting from the primary lesion, along the dermal and subcutaneous lymphatics.

The aim of the present study was to evaluate the frequency of SN in patients from Central Tunisia and to assess the epidemiological and clinical features and the outcome of the disease in the affected patients. 


\section{Materials and methods}

Our study is a retrospective one that includes all CL diagnosed in the laboratory of Parasitology of the Farhat Hached teaching hospital, Sousse, Tunisia, between $1^{\text {st }}$ January 2000 and $31^{\text {th }}$ December 2012. Most of the patients were addressed to the laboratory for suspected CL by the department of Dermatology of the same hospital. Patients' data including age, sex, geographical origin, place of contamination, clinical presentation, treatment and outcome were collected.

The diagnosis of CL was achieved by the demonstration of amastigotes in Giemsa-stained cutaneous smears. Patients with lesions very evocative of CL but found negative on direct examination were further submitted to PCR, known to be more sensitive. Culture on NNN or coagulated rabbit serum (CRS) medium was mainly carried out for the purpose of typing strains either by isoenzymes' electrophoresis or molecular techniques.

\section{Results}

\section{Cutaneous leishmaniasis cases}

Over the 13 year study period, 2128 cases of CL were diagnosed in the laboratory, the majority of them by the demonstration of Leishmania amastigotes in dermal smears. Ninety five per cent of the affected patients originate from one of the following Central Tunisia Governorates (Districts), known to be endemic or highly endemic for ZCL [1, 2]: Sidi Bouzid, Kairouan, Mahdia and Sousse. The 5\% remaining patients originate from Northern or Southern areas or from neighboring countries (Lybia, Algeria). Out of the 2128 cases, 2059 were confirmed (by typing strains) or very likely ZCL cases (according to the geographical origin or place of contamination, seasonal occurrence, large amastigotes on stained smears, outcome, additional cases in families and neighbors). The 96 remaining cases were mostly L. infantum SCL, and few L. killicki CCL. The age of patients ranged from 1 month to 91 years (median 26.8 years). One thousand two hundred thirty four (1234) patients were females and 894 males (sex ratio F/M: 1.38).

Most of the patients presented with single or more often multiple ulcerated lesions located on the limbs, the face and to a lesser extent on the trunk. According to patients' declarations, the lesions appeared 10 days to $>$ one year prior to first examination.

Patients with a few lesions $(<5)$ were treated with intralesional meglumine antimoniate $\left(\right.$ Glucantime $^{\circledR}$ ) and/or cryotherapy. Those with more lesions were treated with intramuscular Glucantime $^{\circledR}$ for 14 to 20 days.

In most treated patients, scarring was obtained in 2 to 8 weeks. In a few of them, however, lesions persisted for a longer period and additional cures were delivered.

2. Cases with sporotrichoid nodules: 
Out of the 2059 patients with ZCL, 34 (1.7\%) developed SN. Fifteen of them were from Mahdia Governorate, 9 from Kairouan Governorate, 6 from Sousse Governorate and 4 from Sidi Bouzid Governorate. It is worth mentioning that Sidi Bouzid region, known to include the most active foci of ZCL in Central Tunisia, was less represented then the 3 remaining Governorates. Inside Governorates, the contamination of all 34 patients took place in areas known to be highly endemic for ZCL.

According to gender, females were more represented then males (23 vs. 11) and F/M sex ratio was higher as compared to common ZCL cases (2.1 vs. 1.4).

The age of patients with sporotrichoid form ranged from 2.5 years to 81 years. Median (47.8 years) was higher as compared to cases without SN (26.8 years).

In $28(82.4 \%)$ patients, the nodules appeared spontaneously a few days to 3 weeks after the onset of the primary lesions. In $5(4.7 \%)$ patients, the SN developed after intralesional antimonial treatment and in one patient after a 3 day cure of intramuscular Glucantime ${ }^{\circledR}$.

In the 34 patients, primary ulcers appeared 2 weeks to 5 months prior to the date of diagnosis (median 2.2 months). Their number ranged from 1 to 12 (median: 3.5). They measured $0.5 \mathrm{~cm}$ to $6 \mathrm{~cm}$; ninety per cent were between $0.5 \mathrm{~cm}$ and $3 \mathrm{~cm}$. They were mainly located on upper limbs: $27.4 \%$ on forearms, $26.2 \%$ on hands and fingers, $11.9 \%$ on arms, $9.5 \%$ on wrists and $4.8 \%$ on elbows. Lower limbs and other sites (nose, neck, eyelids, cheeks, back) were much less involved. Both sides of the body were equally represented.

The number of SN per patient ranged from 2 to $>10$. Most of patients $(76.5 \%)$ had 2 to 4 nodules; 5 had $>10$ nodules.

The size of nodules ranged between $0.5 \mathrm{~cm}$ to $3 \mathrm{~cm}$; the majority of them $(90.7 \%)$ measured $0.5 \% \mathrm{~cm}$ to $1 \mathrm{~cm}$.

Nodules mainly developed on forearms (59.5\%) and arms (23.8\%). Lower limbs and other sites were much less affected. The left side of the body was slightly more represented than the right one $(56.1 \%$ vs. $43.9 \%)$.

In 3 out of the 34 patients, biopsy of nodules was performed. In none of them amastigotes could be demonstrated.

Leishmania strains isolated from 10 out the 34 patients were typed either by enzymes' electrophoresis or by PCR according to Tordini et al. [7]. Nine strains were found to be L. major and one L. killicki.

Whenever SN were observed in patients initially treated with intralesional infiltrations of antimonials, the treatment was switched to the intramuscular form. Thirty three patients responded well to treatment and SN together with primary lesions scared in 2 to 8 weeks. In the last patient, the primary lesions and the nodules persisted up to 13 months. This patient was suffering of scleroderma and submitted to a long-term corticotherapy. In addition, she developed symptoms of antimonial toxicity while receiving intramuscular Glucantime ${ }^{\circledR}$. 


\section{Discussion}

The sporotrichoid form (SF) of cutaneous leishmaniasis is defined by the development of palpable (and often visible) painless subcutaneous nodules distributed in a linear fashion along the lymphatics and extending proximally from a cutaneous lesion. In the Old World, sporotrichoid leishmaniasis has mainly been associated with $L$. major ZCL $[8,9,10,11]$. Its incidence ranges from $6 \%$ in Iran [12], to $10 \%$ in Saudi Arabia [10] and 11 to $22 \%$ in Sudan [8, 9], whereas it seems to be less frequent in other L. major endemic areas like Turkey and Pakistan [13, 14].

In Tunisia, where ZCL is highly endemic, a previous study conducted by Masmoudi et al. [15] showed the SF to be common among patients originating from ZCL endemic areas in Sfax, Sidi Bouzid and Kasserine Governorates, with an incidence of $18 \%$. In our study, which covered a much longer period and a higher number of patients, the incidence was much lower $(1.7 \%)$. These contrasting results are difficult to explain. One of the factors could be a difference in the pathogenicity of infecting Leishmania strains according to the transmission areas. In a study conducted by Gaafar et al. [9] in Sudan and Saudi Arabia, it was shown that the incidence of the SF could be related to the causative L. major zymodeme. Yet, all characterized Tunisian $L$. major strains whatever obtained from patients, animal reservoirs or sand flies were found to belong to the single MON 25 zymodeme [2, 16, 17]. Nevertheless, we may consider that this zymodeme includes strains with a variable virulence, unevenly distributed over the transmission foci. An additional explanation may be the degree of immunization of infected populations, those living in emergent foci (like Sfax and Kasserine) being more susceptible to dissemination than those living in more ancient foci. In our series, nearly the $2 / 3$ of patients with the SF came from Mahdia and Sousse Governorates where the ZCL epidemics occurred much later as compared to Kairouan Governorate (where the outbreak first arose) and Sidi Bouzid Governorate, now considered to include the more active foci in the region $[1,2]$.

The typing of strains obtained from 10 out of our 34 patients showed that most of them ( 9 strains) were, as expected, L. major. Interestingly the last one was L. killicki. This finding is not too surprising as the patient came from Ain Jloula, a recent emerging focus of L. killicki $[2,16]$; and means that L. killicki may be a causal agent of SF.

Histopathologic studies show the subcutaneous nodules to consist of a granulomatous reaction with lymphocytes, plasma cells, macrophages and giant cells $[9,15,18,19]$. They are supposed to represent an immunological reaction against the lymphatic spread of the parasite or its antigens $[10,11]$. The factors that trigger lymphatic dissemination are unclear but some data suggest that the host immune status could influence this dissemination [10, 11]. Indeed, Sadeghian et al. [20] showed that the leishmanin skin test (LST) was negative in $72 \%$ of SF cases and that none was strongly positive in LST positive patients. They concluded that the SF of $\mathrm{CL}$ is at least partially due to a decrease in cellular immunity. However, the role of immunity should be considered with caution because if the immune response is actually involved, it cannot be the sole or the determinant factor as in this situation the age of patients with SF is expected to be lower than that of those with the common ZCL form, the children being much more affected. Yet, this is not the case in our study neither in that of Masmoudi et al. [15]. where 
the median age of patients with SF was higher as compared to patients with the common form (47.8 years and 26.8 years respectively).

In our study, females were more represented then males $(\mathrm{F} / \mathrm{M}$ sex ratio $=2.1)$. A similar finding $(\mathrm{F} / \mathrm{M}$ sex ratio $=1.7)$ was reported by Masmoudi et al. [15]; whereas in other previous studies, no significant association of subcutaneous nodules with sex was reported [9].

The time to appearance of nodules is variable. It ranges from a few days to a few weeks or months; nodules may even appear after complete healing of the primary cutaneous lesions [8], with a delay reaching 1 or 2 years in some reported cases [15, 21]. In most of our patients, the nodules appeared in less than one month after the onset of the primary lesions.

Among the factors that trigger the lymphatic dissemination of parasites away from the primary lesions, many reports suggest the intralesional antimonial treatment to play a role $[9,10,11$, 22]. The reason for that is unclear. Nevertheless, this factor appears not to be determinant as only 6 out of our 34 patients $(17.6 \%)$ had received intralesional Glucantime ${ }^{\circledR}$; in the remaining 28 patients the nodules appeared spontaneously. The same finding was reported in Masmoudi et al. study [15] where $74 \%$ of patients did not receive any Glucantime ${ }^{\circledR}$ infiltrations before the development of nodules.

The number of sporotrichroïd nodules is reported to be variable, but they often are multiple $[10,11,19,21]$. In our patients the number ranged from 2 to $>10$; most of patients had 2 to 4 nodules as shown in figure 1. In the series of Masmoudi et al. [15], this number reached 20 nodules in one patient, with a mean of 7 nodules.

When mentioned, the size of nodules is usually 1 to $2 \mathrm{~cm}[11,15,19]$. In our patients, most nodules sized between 0.5 to $1 \mathrm{~cm}$, the largest being $3 \mathrm{~cm}$.

In our 34 patients, nodules mainly developed on forearms and arms; the lower limbs and other sites of the body were much less represented. This finding was expected as the distribution of nodules overall reflects that of primary lesions which mainly involved the upper limbs. This result is very similar to that of Masmoudi et al. [15] where nodules were located on upper limbs in $80 \%$ of cases, together with the primary ulcers. Nevertheless, other sites can be affected; indeed, in some of our patients, SN developed on the legs, the cheeks, the eyelids, the nose and the back.

The diagnosis of CL in all of our 34 patients was confirmed by the demonstration of amastigotes in ulcerated primary lesions, while SF was diagnosed on the basis of clinical criteria. In the 3 patients where the biopsy of nodules was performed, no parasites could be shown. According to previous reports, amastigotes may or may not be demonstrable on smears or impressed biopsies nodules $[8,10,15,19,23]$. The diagnosis of the SF is usually suspected when nodules develop in the vicinity of a previously confirmed primary CL lesion, and resolve under antimonial treatment. As stated above, the amastigotes may or may not be demonstrated in SN. Similar conditions mainly include sporotrichosis that, to our knowledge, has never been reported in Tunisia. Cutaneous tuberculosis and atypical mycobacteriosis can also be considered but both conditions are unresponsive to antimonials. 


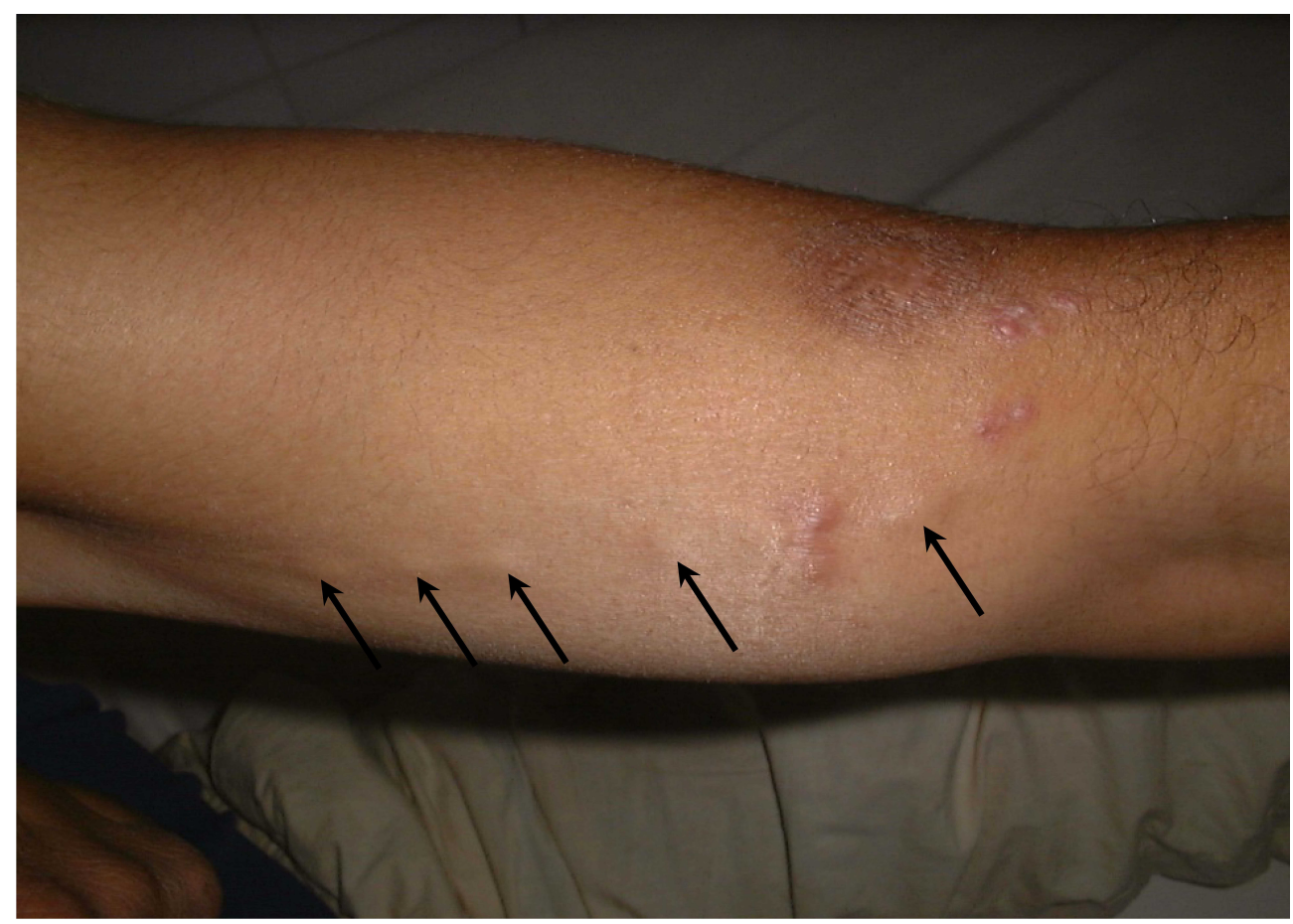

Figure 1. Sporotrichoid nodules (arrows) on the forearm of a patient.

While in localized CL local treatment such as intralesional infiltrations of pentavalent antimonials and /or cryotherapy may be helpful and sufficient, such treatment is of no value in disseminated disease where systemic antimonials must be started [23, 24]. In most reported patients with SF, systemic treatment was successful and outcome favorable [5, 14, 15], even though some patients were reported to be unresponsive to antimonials [11, 19, 22]. Thirty three $(97 \%)$ of our patients responded well to systemic Glucantime ${ }^{\circledR}$ and SN together with primary lesions scared in 2 to 8 weeks. In the last patient, both primary ulcers and SN persisted up to 13 months. It is worth mentioning that this patient was receiving a long term corticotherapy and developed symptoms of antimonials toxicity which led to the interruption of the Glucantime ${ }^{\circledR}$ cure.

\section{Conclusion}

The SF of CL appears to be a rather rare event in Central Tunisian SCL foci. In patients affected, outcome is similar to uncomplicated cases. 


\section{Author details}

Moncef Ben Saïd ${ }^{1 *}$, Fatma Saghrouni ${ }^{1}$, Yusr Saadi-Ben Aoun ${ }^{2}$, Najet Ghariani ${ }^{3}$, Alia Yaacoub ${ }^{1}$, Henda Ach ${ }^{1}$, Mohamed Denguezli ${ }^{3}$, Ikram Guizani ${ }^{2}$ and Akila Fathallah-Mili ${ }^{1}$

*Address all correspondence to: moncef.bensaid@rns.tn

1 Laboratory of Parasitology, Farhat Hached Hospital, Sousse, Tunisia

2 Laboratory of Parasitic Epidemiology and Ecology (LEEP)-LR00SP04, Institut Pasteur, Tunisia

3 Service of Dermatology, Farhat Hached Hospital, Sousse, Tunisia

\section{References}

[1] Ben Rachid, M.S., Ben Ismail, R. \& Ben Said, M. The ecology of visceral and cutaneous leishmaniasis in Tunisia. In. "Proceedings of the international workshop on leishmaniasis control strategies", 25-29 nov. 1991, Merida, Mexico, IDRC and UPCH eds. (1992), pp. 131-154.

[2] Fathallah, A., Saghrouni, F. Ben Saïd, Z., Saadi-Ben Aoun, Y., Guizani, I. \& Ben Saïd, M. Retrospective analysis of leishmaniasis in Central Tunisia : An update on emerging epidemiological trends. In: Current Topics in Tropical Medicine, 2012, Dr. Alfonso Rodriguez Morales (Ed.), ISBN: 978-953-51-0274-8, In Tech, chp.15, pp: 228-252.

[3] Chaffai, M, Ben Rachid, M.S., Ben Ismail, R., Ben Osman, A. \& Makni, N. Clinico-epidemiological forms of cutaneous leishmaniasis in Tunisia. Ann Dermatol venereol. (1988), 115: 1255-1260.

[4] Bryceson, A. D. M. Tropical dermatology: Cutaneous leishmaniasis. Br J Dermatol. (1976), 94: 223-226.

[5] Lopez-Escobar, M., Drake-Monfort, M., Salesa-Gutierrez de Rosas, R. \& HermanaRamirez, S. Sporotrichoid cutaneous leishmaniasis. Actas Dermo Sifiliogr. (2007), 98: 441-445.

[6] Spier, S., Medenica, M., McMillan, S. \& Virtue, C. Sporotrichoid leishmaniasis. Arch Dermatol. (1977), 113: 1104-1105.

[7] Tordini, G., Giaccherini, R., Pacenti, L., Miracco, C., Zazzi, M., \& Zanelli, G. Cutaneous leishmaniasis: usefulness of PCR on paraffin-embedded skin biopsies as part of routine investigation. Ann Trop Med Parasitol. (2007), 101: 745-749. 
[8] El-Safi, S.H., Peters, W., El -Toam,B., El-kadarow, A. \& Evans, D. A. Studies on the leishmaniases in the Sudan. 2. Clinical and parasitological studies on cutaneous leishmaniasis. Trans R Soc Trop Med Hyg. (1991), 85: 457-464.

[9] Gaafar, A., Fadl, A., El Kadaro, A.Y., El Hassan, M.M., Kemp, M., Ismail, A.I.A., Morgos, S.A. \& El Hassen A.M. Sporotrichoid cutaneous leishmaniasis due to Leishmania major of different zymodemes in the Sudan and Saudi Arabia: a comparative study. Trans R Soc Trop Med Hyg. (1994), 88: 552-554.

[10] Kubba, R., El Hassan, A.M., Al-Gindan, Y., Omer, A.H., Kutty, M.K. \& Saeed, M.B. Dissemination in cutaneous leishmaniasis. II-Satellite papules and subcutaneous induration. Int J Dermatol. (1988), 27: 702-706.

[11] Morelli, P., Gianelli, E., Calattini, S., Corbellino,M., Antinori, S. \& Meroni, L. Itraconazole can be effective in the treatment of sporotrichoid leishmaniasis. J Travel Med. (2004); 11: 328-330.

[12] Talari, S.A., Talaei, R., Shajari, G., Vakili, Z. \&Taghaviardakani A. Childhood cutaneous leishmaniasis: report of 117 cases from Iran. Korean J Parasitol. (2006), 44: 355-360.

[13] Gülru Erdogan, F., Gül Çakir, A., Gököz, ö. \& Gürler, A. A case of sporotrichoid cutaneous leishmaniasis. Turkderm (2011), 45: 100-103.

[14] Iftikhar, N., Bari, I. \& Ejaz, A. Rare variants of cutaneous leishmaniasis: whitlow, paronychia, and sporotrichoid. Int J Dermatol. (2003), 42: 807-809.

[15] Masmoudi, A., Ayadi, N., Khabir, A., Bouzid, L., Bouassida, S., Meziou, T.J., Akrout, F., Zahaf, A., Boudawara, T. \& Turki, H. Sporotrichoid cutaneous leishmaniasis in Tunisia: a clinical and histological study. Ann Dermatol Venereol. (2008), 135:63-67.

[16] Aoun, K., Amri, F., Chouihi, E., Haouas, N., Bedoui, K., Ben Khelef, R. \& Bouratbine A. Epidemiology of Leishmania $(L)$ infantum, L. major and L. killicki in Tunisia : results and analysis of the identification of 226 human and canine isolates. Bull Soc Pathol Exot. (2008), 101: 323-328.

[17] Haouas, N., Gorcii, M., Chargui, N., Aoun, K., Bouratbine, A., Messaadi-Akrout, F., Masmoudi, A., Zili, J., Ben Saïd, M., Pratlong, F., Dedet, J.P., Mezhoud, H., Azaiez, R. \& Babba, H. Leishmaniasis in central and southern Tunisia: current geographical distribution of zymodemes. Parasite (2007); 14: 239-246.

[18] Kibbi, A.G., Karam, P.G. \&Kurban, A.K. Sporotrichoid leishmaniasis in patients from Saudi Arabia: clinical and histological features. J Am Acad Dermatol. (1987), 17: 759-764.

[19] Neva, F.A., Wyler, D. \& Nash, T. Cutaneous leishmaniasis - A case with persistent organisms after treatment in presence of normal immune response. Am J Trop Med Hyg. (1979), 28: 467-471. 
[20] Sadeghian, G., Ziaei, H., Bidabadi, L.S. \& Nilforoushazdeh, M.A. Evaluation of leishmanin skin test reaction in different variants of cutaneous leishmaniasis. Int J Dermatol. (2013), 58: 239.

[21] Ejaz, A., Raza, N. \& Iftikhar, N. Recurrent cutaneous leishmaniasis presenting as sporotrichoid abscesses: a rare presentation near Afghanistan border. Dermatology Online Journal (2007), 13: 15.

[22] Cozzani, E., Satta, R., Fausti, V., Cottoni, F. \& Parodi A. Cutaneous sporotrichoid leishmaniasis resistant to pentavalent antimonial therapy: complete resolution with itraconazole. Clin Exp Dermatol. (2011), 36: 49-51.

[23] Tobin, E.H. \& Jih, W.W. Sporotrichoid lymphocutaneous infections: etiology, diagnosis and therapy. Am Fam Physician. (2001), 63: 326-332.

[24] Killick-Kendrick, R. \& Peters, W. Leishmaniasis in Arabia: an annotated bibliography. Am J Trop Med Hyg. (1991), 44, supplement p.31. 
